\title{
Energy Breakdown in Capacitive Deionization
}

\author{
Ali Hemmatifar, ${ }^{\mathrm{a}}$ James W. Palko, ${ }^{\mathrm{a}}$ Michael Stadermann, ${ }^{\mathrm{b}}$ and Juan G. Santiago ${ }^{\mathrm{a}, *}$
}

${ }^{a}$ Department of Mechanical Engineering, Stanford University, Stanford, California 94305, United States

${ }^{\mathrm{b}}$ Lawrence Livermore National Laboratory, 7000 East Avenue, Livermore, California 94550, United States

* To whom correspondence should be addressed. Tel. 650-736-1283, Fax 650-723-7657, E-mail: juan.santiago@stanford.edu

\begin{abstract}
We explored the energy loss mechanisms in capacitive deionization (CDI). We hypothesize that resistive and parasitic losses are two main sources of energy losses. We measured contribution from each loss mechanism in water desalination with constant current $(\mathrm{CC})$ charge/discharge cycling. Resistive energy loss is expected to dominate in high current charging cases, as it increases approximately linearly with current for fixed charge transfer (resistive power loss scales as square of current and charging time scales as inverse of current). On the other hand, parasitic loss is dominant in low current cases, as the electrodes spend more time at higher voltages. We built a CDI cell with five electrode pairs and standard flow between architecture. We performed a series of experiments with various cycling currents and cut-off voltages (voltage at which current is reversed) and studied these energy losses. To this end, we measured series resistance of the cell
\end{abstract}


(contact resistances, resistance of wires, and resistance of solution in spacers) during charging and discharging from voltage response of a small amplitude AC current signal added to the underlying cycling current. We performed a separate set of experiments to quantify parasitic (or leakage) current of the cell versus cell voltage. We then used these data to estimate parasitic losses under the assumption that leakage current is primarily voltage (and not current) dependent. Our results confirmed that resistive and parasitic losses respectively dominate in the limit of high and low currents. We also measured salt adsorption and report energy-normalized adsorbed salt ( ENAS, energy loss per ion removed) and average salt adsorption rate ( $A S A R)$. We show a clear tradeoff between $A S A R$ and ENAS and show that balancing these losses leads to optimal energy efficiency.

Keywords: Capacitive deionization, Water desalination, Energy consumption, Porous carbon electrodes, Performance optimization

\section{Nomenclature}

\begin{tabular}{|lll|}
\hline Parameter & Description & Unit \\
$E_{\text {in }}$ & Energy input to the cell during charging & $\mathrm{J}$ \\
$E_{\text {out }}$ & Energy recovered during discharging & $\mathrm{J}$ \\
$E_{\text {in }}^{R}$ & Resistive energy loss during charging & $\mathrm{J}$ \\
$E_{\text {out }}^{R}$ & Resistive energy loss during discharging & $\mathrm{J}$ \\
\hline
\end{tabular}




\begin{tabular}{|c|c|c|}
\hline$E_{\text {in }}^{R, S}$ & Resistive loss due to series resistances during charging & $\mathrm{J}$ \\
\hline$E_{\text {out }}^{R, S}$ & Resistive loss due to series resistances during discharging & $\mathrm{J}$ \\
\hline$E_{i n}^{R, N S}$ & \multicolumn{2}{|c|}{ Resistive loss due to distributed, non-series resistance inside electrode pores } \\
\hline \multicolumn{2}{|c|}{ during charging } & $\mathrm{J}$ \\
\hline$E_{\text {out }}^{R, N S}$ & \multicolumn{2}{|c|}{ Resistive loss due to distributed, non-series resistance inside electrode pores } \\
\hline \multicolumn{2}{|c|}{ during discharging } & $\mathrm{J}$ \\
\hline$E_{i n}^{P}$ & Parasitic energy loss during charging & $\mathrm{J}$ \\
\hline$E_{\text {out }}^{P}$ & Parasitic energy loss during discharging & $\mathrm{J}$ \\
\hline$E_{c a p}$ & Stored energy in the cell & $\mathrm{J}$ \\
\hline$V_{e x t}$ & External voltage measured via sourcemeter & $\mathrm{V}$ \\
\hline$V_{\max }$ & Maximum allowable external voltage & $\mathrm{V}$ \\
\hline$V_{c a p}$ & Equivalent capacitance voltage, $V_{e x t} \pm I_{0} R_{s}(t)$ & $\mathrm{V}$ \\
\hline$\Delta V_{c a p}$ & Maximum range of $V_{\text {cap }}$, defined as $V_{\text {cap }, \text { max }}-V_{\text {cap, min }}$ & $\mathrm{V}$ \\
\hline$I_{0}$ & External current magnitude applied to the cell & $\mathrm{mA}$ \\
\hline C & CDI cell capacitance & $\mathrm{F}$ \\
\hline$R_{s}$ & Series resistance, including wires, interfacial electrode-cur & collector \\
\hline \multicolumn{2}{|c|}{ resistance, and solution resistance in spacer and pores } & $\Omega$ \\
\hline$R_{p}$ & Parallel resistance responsible for parasitic losses & $\Omega$ \\
\hline$t_{\text {charge }}$ & Charging time & $\mathrm{s}$ \\
\hline$t_{c y c l e}$ & Cycle time & $\mathrm{s}$ \\
\hline
\end{tabular}




\begin{tabular}{|lll|}
\hline$\tau_{R C}$ & RC time scale of the cell & $\mathrm{s}$ \\
$c_{0}$ & Influent salt concentration & $\mathrm{mM}$ \\
$Q$ & Flow rate & $\mathrm{ml} \mathrm{min}^{-1}$ \\
$\Gamma_{\text {ads }}$ & Salt adsorption during charging & $\mu \mathrm{mole}$ \\
$A S A R$ & Average salt adsorption rate $\left(\Gamma_{\text {ads }} / N A t_{\text {cycle }}\right)$ & $\mu$ mole $\mathrm{cm}^{-2} \mathrm{~min}^{-1}$ \\
$E N A S$ & Energy-normalized adsorbed salt $\left(\Gamma_{\text {ads }} /\left(E_{\text {in }}-E_{\text {out }}\right)\right)$ & $\mu$ mole J ${ }^{-1}$ \\
\hline
\end{tabular}

\section{Introduction}

Energy has traditionally been the dominant cost component for many desalination systems such as those applying distillation, which is highly energy intensive (Anderson et al., 2010). Reverse osmosis (RO) has dramatically reduced the energy requirements for desalination, with modern systems achieving roughly 50\% energy efficiency for treating seawater based on the thermodynamic ideal free energy of mixing (Elimelech and Phillip, 2011). However, RO fares significantly worse for water with lower concentrations of dissolved solids, such as brackish water, where it only reaches $10 \%$ or less efficiency (Shrivastava et al., 2014). RO forces all treated water through the active membrane, with energy losses (and plant size) roughly corresponding to the total throughput of the plant.

Capacitive deionization (CDI) is a method of desalination that directly acts on the ions in solution and sequesters them into electric double layers leaving purified water, which is flushed from the cell. CDI has been investigated in various forms for over 50 years (Blair and Murphy, 1960; Johnson and Newman, 1971), but has recently seen a rapid increase in activity. Because the 
ions themselves are directly targeted, the energy consumption of this technique largely scales with the amount of salt removed (i.e. throughput times input concentration). This scaling promises higher energy efficiency for CDI compared to competing technologies when treating waters with lower dissolved solid concentrations than seawater (e.g. brackish water) (Zhao et al., 2013). There are a variety of operational parameters that can be tuned for CDI, including time dependence of charging voltage or current, level of cell charging (i.e. final cell voltage), and flow rate. The choice of these can dramatically influence the energy efficiency achieved in operation, and a consistent framework for determining optimal conditions for operation of CDI cells is still lacking.

We note that electric double layer capacitors, or supercapacitors, rely on very similar physics to CDI and have been optimized to maximize charge/discharge cycle efficiency and energy storage density. A number of studies have looked at the loss mechanisms present in supercapacitors (Conway, 2013), including series resistance (Conway and Pell, 2002; Yang and Zhang, 2013), charge redistribution loss, and parasitic reaction loss (Ike et al., 2016). However, the design and operational regimes of supercapacitors are very different than CDI. Importantly, there is generally no electrolyte flow, and organic solvent based, high concentration electrolytes are commonly used to achieve high operating voltage windows and minimize resistance. The goal of supercapacitor operation is solely the storage and recovery of energy. Further, supercapacitors are often applied in high current applications, and this requires a focus on series resistive losses. This focus has led to substantial supercapacitor optimization and sub-milliohm equivalent series resistances are commonly achieved (Yu et al., 2013).

The promise of CDI for energy efficient processing of lower concentration inlet feeds has led to a number of studies concerning energy loss (Alvarez-Gonzalez et al., 2016; Choi, 2015; Demirer et al., 2013; J.E. Dykstra et al., 2016; García-quismondo et al., 2015; García-Quismondo 
et al., 2013; Kang et al., 2014; R. Zhao et al., 2012). These have generally focused on the total energy loss of the process, which is useful for comparison with different technologies or among different CDI designs, but provides little insight for optimizing CDI operation or refining current CDI designs. One element that has been studied in some detail is the choice of operation of CDI cells with constant current charging versus constant voltage charging (Choi, 2015; Kang et al., 2014; R. Zhao et al., 2012). Constant current operation generally leads to superior energy performance with energy usage reduced by up to $30 \%$ (Kang et al., 2014)., and some studies have dealt with the specific mechanisms of loss operative in CDI. Alvarez-Gonzalez et al. (2016) developed a simple model accounting for resistive and parasitic losses consisting of series and parallel resistances and parameterized this model using experimental data. They then optimized cell geometry and charging current in terms of cell energy loss using this model and showed good agreement with experiments. Detailed studies have also been conducted on the series resistance of CDI cells, e.g. ( $\mathrm{Qu}$ et al., 2015). Improved understanding of the constituent energy loss mechanisms in CDI offers the opportunity for more efficient operation of existing cells and improved future designs, and hence, motivates this work.

Here, we experimentally quantify the specific energy loss mechanisms operative during CDI with constant current charging. These mechanisms separate roughly into those dominant at high or low charging currents. The mechanisms dominant at high currents motivate slow charging of the cell. We attribute these losses mostly to resistive dissipation during charge and discharge and, to a lesser degree, redistribution of accumulated charge within electrodes. We perform in situ, real-time measurements of cell series resistance as a function of charging current and time within the charging phase. The dominant losses at low charging currents, corresponding to parasitic currents in the cell, prompt acceleration of the charge phase and a reduction of charge time. We 
perform an independent set of constant voltage experiments to measure parasitic currents vs. cell voltage. We characterize both loss categories over a broad operational parameter space and show that balancing these losses leads to optimal energy efficiency. Total salt removed per cycle is another key parameter for CDI operation. We define two figures of merit (FOMs) relevant for practical CDI operation and plant design, salt removed per unit time and salt removed per unit energy. These provide quantitative metrics for evaluating tradeoffs between operational requirements (e.g. throughput vs. energy efficiency). We also provide relations for the investigated CDI cell identifying regimes of charging current and maximum cell voltage which allow a balance between cell throughput and energy efficiency as quantified by the product of salt removal rate and salt removed per unit energy.

\section{Materials and methods}

\subsection{CDI cell design}

Fig. 1a shows a schematic of our radial flow-between CDI (fbCDI) cell. We fabricated the cell using five pairs of activated carbon electrodes (two of which are shown here) with $6 \mathrm{~cm}$ diameter and $270 \mu \mathrm{m}$ thickness and total dry mass of $4.3 \mathrm{~g}$. The electrodes are based on materials provided by Voltea B.V. (Sassenheim, The Netherlands) and consist of commercially available YP-50 activated carbon powder (Kuraray Chemical, Japan), a PTFE binder, and carbon black. The same electrode material has been used and characterized for CDI applications extensively (Biesheuvel, 2015; Biesheuvel et al., 2016; J. E. Dykstra et al., 2016; R Zhao et al., 2012). We stacked the electrodes between $130 \mu \mathrm{m}$ thick circular shaped titanium sheets, which acted as current collectors (total of six sheets). Each current collector had a tab section $(1 \times 5 \mathrm{~cm})$ for connection to external 
wires (c.f. Fig. 1a). All the electrodes and current collectors (except the "book end" electrode and current collector on top of the stack) had a $5 \mathrm{~mm}$ diameter opening at their center for the flow passage. We used $420 \mu \mathrm{m}$ thick non-conductive polypropylene mesh (McMaster-Carr, Los Angeles, CA) between each electrode pair as spacers. We cut the spacers in circles slightly larger ( 4 mm) than electrodes and current collectors to prevent electrical short circuit. This assembly was then housed inside a CNC-machined acrylic clamshell structure and sealed with O-ring gaskets and fasteners (not shown here). Flow paths are indicated with arrows in Fig. 1a. Feed water enters the cell via a $5 \mathrm{~mm}$ diameter inlet port in the upper clamshell and is radially distributed to the outer surfaces of the stack within the header. Feed solution then flows radially inward (toward the center of the stack) through the spacers and between the electrodes. This radial flow empties down into the vertical flow channel and exits via an outlet port in the lower clamshell.

\subsection{Energy pathway in CDI}

The schematic of Fig. 1b shows the energy pathway in a typical CDI cell. To understand this, we first note that the goal in any CDI system is to increase the potential energy of electrode stack from its base level, and consequently, attract ionic species to the electrodes with electrostatic forces. This is done by transferring electrons to the cell through an external voltage and/or current source. This input energy is denoted as $E_{i n}$ in Fig. 1b. However, not all transferred potential energy is used for ionic charge storage (capacitive energy, or $E_{c a p}$ ), as there are various loss mechanisms during the charging process. Namely, resistive and parasitic energy losses, denoted respectively as $E_{i n}^{R}$ and $E_{i n}^{P}$ in Fig. 1b. The charging process continues until one or more charging criteria are met, such as a specified maximum cell voltage or a pre-set amount of transferred electronic charge. Then the regeneration or discharge process starts and gradually lowers the stack's potential energy 
level to its base level. The extractable or recoverable energy $\left(E_{\text {out }}\right)$, however, is smaller than $E_{\text {cap }}$, as there are resistive and parasitic energy losses in discharge process as well ( $E_{\text {out }}^{R}$ and $E_{\text {out }}^{P}$ respectively).

We emphasize that $E_{i n}$ is the total electrical energy input during charging. We measure $E_{i n}$ as the (unsigned) magnitude area under the voltage versus time curve during charging multiplied by the current during charging. As we shall describe, we measure $E_{\text {out }}$ as the (unsigned) magnitude area under the voltage versus time curve during discharge multiplied by the current during discharge. A portion of $E_{i n}$ is dissipated (by internal resistance and parasitic reaction losses) and the rest of $E_{i n}$ is stored as capacitive energy. The energy loss in the entire charge and discharge phase is thus equal to $E_{\text {in }}-E_{\text {out }}$. The following two equations can then describe the energy pathway in CDI systems.

$$
\begin{gathered}
E_{\text {in }}-E_{\text {out }}=\left(E_{\text {in }}^{R}+E_{\text {out }}^{R}\right)+\left(E_{\text {in }}^{P}+E_{\text {out }}^{P}\right) \\
E_{\text {cap }}=E_{\text {in }}-\left(E_{\text {in }}^{R}+E_{\text {in }}^{P}\right)
\end{gathered}
$$

We further define resistive loss during charging and discharging as

$$
\begin{aligned}
& E_{\text {in }}^{R}=E_{\text {in }}^{R, N S}+E_{\text {in }}^{R, S}=E_{\text {in }}^{R, N S}+\int_{0}^{t_{\text {thange }}} I_{0}^{2} R_{s}(t) \mathrm{d} t, \\
& E_{\text {out }}^{R}=E_{\text {out }}^{R, N S}+E_{\text {out }}^{R, S}=E_{\text {out }}^{R, N S}+\int_{t_{\text {chunge }}}^{t_{\text {cove }}} I_{0}^{2} R_{s}(t) \mathrm{d} t,
\end{aligned}
$$

where $E_{i n}^{R, S}$ and $E_{\text {out }}^{R, S}$ are series resistive loss during charging and discharging, respectively. Series resistance here corresponds to contact resistance, ionic resistance of solution in separators, and resistance of wires. Similarly, $E_{i n}^{R, N S}$ and $E_{\text {out }}^{R, N S}$ are energy loss due to network of distributed ionic resistance of solution inside the electrode pores during charging and discharging. Superscript NS stands for non-series resistance. We here will neglect the resistances of the electrode matrix as this 
tends to be negligible in CDI (e.g., compared to ionic resistance in electrodes) (J. E. Dykstra et al., 2016; Qu et al., 2015).

We separated resistive loss contributions into series and non-series resistances because of their distinct behavior, as described in the following. The equivalent circuit of a CDI cell can be described as a network of resistors and non-linear capacitors (Qu et al., 2015; Suss et al., 2013). Some of these resistors are electrically in series and others are parallel to capacitors. The series resistors include the external lead resistances, the current collector, and the non-series resistance associated with the electrolyte inside the pores of the (porous dielectric) spacers. The voltage (current) response of these series resistors to rapid changes in current (voltage) can be assumed to be instantaneous. In contrast, the distributed resistor/capacitor network of the porous CDI cell electrodes have significant characteristic RC (resistance-capacitance) time delays associated with charging (order 10's of seconds or greater for significant penetration of charge into the electrode). As a result, due to its fast time response, series resistances can be measured at each time during charging and discharging (c.f. Section 3.2), while it is not feasible to directly measure values of $E_{i n}^{R, N S}$ and $E_{\text {out }}^{R, N S}$ in situ and independently. We therefore directly measure series resistive loss (c.f. Section 3.2) and also quantify parasitic loss with a separate experiment. We then use Eq. (1) to calculate the sum of non-series resistive loss for the charging and discharge phases $\left(E_{\text {in }}^{R, N S}+E_{\text {out }}^{R, N S}\right.$ ). In this paper, we perform a series of experiments to distinguish contribution of different loss mechanisms (resistive and parasitic mechanisms) and study energetic performance in CDI. 

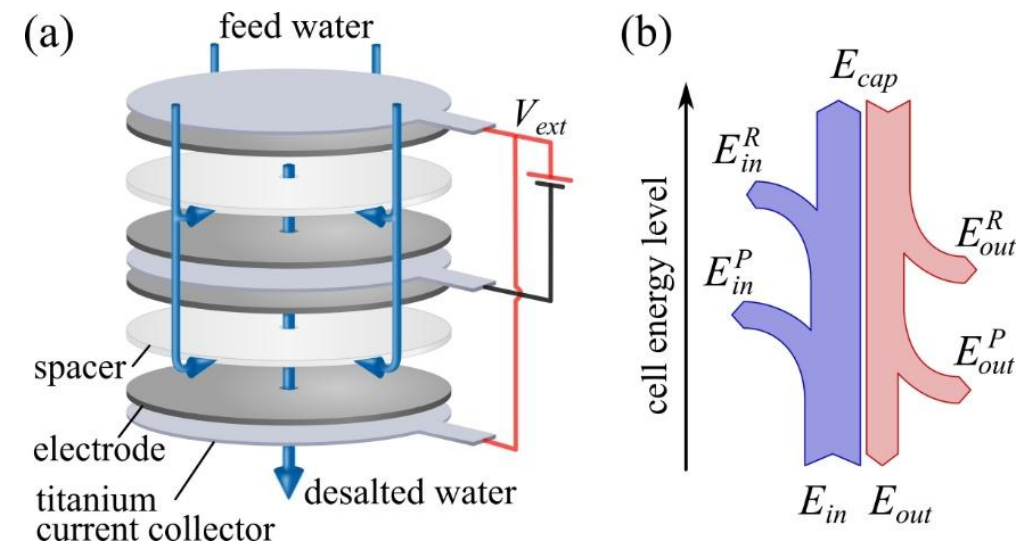

Fig. 1. (a) Schematic of circular fbCDI cell with five pairs of activated carbon electrodes (only two pairs shown here). The stack was housed inside a clamshell structure (not shown here) and sealed with O-rings and fasteners. Arrows indicate flow paths. (b) Schematic of energy pathway in a typical CDI system. A fraction of input energy $E_{i n}$ during charging is dissipated via resistive ( $E_{\text {in }}^{R}$ ) and parasitic $\left(E_{i n}^{P}\right)$ processes and the rest is stored in the cell ( $\left.E_{c a p}\right)$. A portion of stored energy is then dissipated during discharging ( $E_{\text {out }}^{R}$ and $\left.E_{\text {out }}^{P}\right)$ and remaining energy is recovered $\left(E_{\text {out }}\right)$.

\subsection{Experimental procedure}

The experimental setup consisted of our fbCDI cell (c.f. Section 2.2), a 3 L reservoir filled with $50 \mathrm{mM}$ potassium chloride $(\mathrm{KCl})$ solution, a peristaltic pump (Watson Marlow 120U/DV, Falmouth, Cornwall, UK), a sourcemeter (Keithley 2400, Cleveland, OH), and a flow-through conductivity sensor (eDAQ, Denistone East, Australia). We used $\mathrm{KCl}$ to approximate a univalent, binary, and symmetric solution. We operated our cell at constant current (CC) charging and discharging to study the energy budget introduced in Section 2.2. We used a fixed flow rate of $2 \mathrm{~mL} \mathrm{~min}^{-1}$ with closed-loop circulation in all of our experiments (flow from reservoir to cell and back to reservoir). This is equivalent to normalized flow rate of $0.014 \mathrm{~mL} \mathrm{~min}^{-1} \mathrm{~cm}^{-2}$ (flow rate 
divided by stack electrode area, $N A$, where $N=5$ is number for electrode pairs and $A \approx 28 \mathrm{~cm}^{2}$ is single electrode area). We continuously purged the reservoir with high purity argon gas during the experiments. We estimate $<1 \%$ change in reservoir concentration based on adsorption capacity of our cell, and approximate influent concentration as constant in time. We estimate a flush time (defined here as the time to replace one cell volume) of about $3 \mathrm{~min}$. We applied external currents of $25,50,100,150,200$, and $300 \mathrm{~mA}$ in charging and a reversed current of the same magnitude in the discharging process. These values are equivalent to current densities of 1.8, 3.6, 7.1, 10.7, 14.2, and $21.4 \mathrm{~A} \mathrm{~m}^{-2}$ (current divided by stack electrode area, $N A$ ). For each current, we charged the cell to fixed external voltage values between 0.2 to $1.2 \mathrm{~V}$ (with $0.2 \mathrm{~V}$ increments) and discharged the cell to $0 \mathrm{~V}$. Higher currents had necessarily narrower working voltage because of considerable resistive voltage drop. For example, we charged the cell to $0.6,0.8,1,1.2 \mathrm{~V}$ at highest $300 \mathrm{~mA}$ current. For each external current and voltage combination (total of 32 experiments), we performed at least three complete charge/discharge phases. This ensured the dynamic steady state (DSS) condition, in which salt adsorption during charging is equal to desorption during discharging. DSS was reached after a few cycles, and voltage and effluent concentration profiles did not vary between cycles thereafter. As shown by Cohen et al. (2015), salt removal performance of CDI cells can be prone to degradation under prolonged experiments. This is believed to be partly due to oxidation and corrosion of positive electrode. We did not observe noticeable degradation during the course of our experiments (which were performed over a period of about 2 months).

We recorded external voltage and effluent conductivity using a Keithley sourcemeter and an eDAQ conductivity sensor (with $\sim 93 \mu \mathrm{L}$ internal channel volume). Conductivity was converted to salt concentration using a calibration curve for KCl. Refer to Section S.1 and S.2 of Supplementary Information (SI) for plots of voltage and concentration measurements for the 
experimental conditions mentioned above. We also show the establishment of DSS condition for the case of $100 \mathrm{~mA}$ current and $0.8 \mathrm{~V}$ maximum voltage in Section S.2 of the SI. Additionally, in order to estimate resistive losses, we used a sourcemeter for in-situ series resistance measurement during charging and discharging. See Section 3.2 and Section S.3 of the SI for more details.

\section{Results and discussion}

\subsection{Voltage profile and energy breakdown}

Fig. 2a shows voltage profiles of our cell vs. time with $200 \mathrm{~mA}$ charge/discharge current and limit voltage of $V_{\max }=1.2 \mathrm{~V}$ and $2 \mathrm{~mL} \mathrm{m^{-1 }}$ flow rate (under DSS condition). Solid curve shows voltage measured by the sourcemeter and denoted as $V_{\text {ext }}$. Dashed curve corresponds to underlying “equivalent capacitance" voltage $\left(V_{\text {cap }}\right)$, the total voltage difference across the electrodes excluding voltage drop across the series resistance. We term this $V_{c a p}$ as an analogy to the equivalent RC circuit shown as an inset in Fig. 2a and we define it as $V_{e x t}-I_{0} R_{s}$ or $V_{e x t}+I_{0} R_{s}$ respectively during charging and discharging ( $R_{s}$ and $I_{0}$ being series resistance and external current magnitude). The instantaneous rise/drop in $V_{\text {ext }}$ shown in Fig. 2a is because of series resistance and is equal to $2 I_{0} R_{s}$ . The prefactor 2 is consistent with the reversal of current at the start of charging or discharging. $V_{\text {cap }}$ also exhibits a small, abrupt drop after current reversal as well. We hypothesize the latter effect is due to charge redistribution in the porous carbon electrodes, which has been observed in transmission line (Black and Andreas, 2010, 2009) and high-fidelity models (Hemmatifar et al.,

2015; Rica et al., 2013) of CDI as well as in experiments (Długołęcki and van der Wal, 2013; Pell 
et al., 2000). Refer to Fig. S.1 of the SI for plots of voltage measurements at other experimental conditions.

In Fig. 2b, we show power input/generation of our fbCDI cell under the same conditions as those of Fig. 2a. This plot is generated by multiplying $V_{\text {ext }}$ and $V_{\text {cap }}$ by external current $I_{0}$. Positive $I_{0} V_{e x t}$ values correspond to power transferred to the cell and negative $-I_{0} V_{\text {ext }}$ values are power generated by the cell—-power which can ideally (in the limit of perfect transfer efficiency) be stored or used. Shaded regions show total input $\left(E_{\text {in }}\right)$ and output (recovered) energy $\left(E_{\text {out }}\right)$ of the cell. Diagonal and vertical hatched areas are respectively measured series resistive loss $\left(E_{i n}^{R, S}\right.$ and $\left.E_{\text {out }}^{R, S}\right)$ and parasitic loss $\left(E_{\text {in }}^{P}\right.$ and $E_{\text {out }}^{P}$ ) during charging and discharging. We calculated series resistive loss using in-situ, in-line measurement of series resistance (c.f. Section 3.2 for more information). Further, we measured parasitic energy loss through an independent set of constant voltage experiments. We hypothesize that the parasitic loss is primarily due to leakage currents associated with Faradaic reactions at electrodes. To this end, we charged the cell to fixed external voltage values between 0.1 to $1.2 \mathrm{~V}$ (with $0.1 \mathrm{~V}$ increments) for $25 \mathrm{~min}$ each and monitored the current via sourcemeter. We attribute the remaining current at $25 \min \left(>10 \tau_{R C}\right.$, with $\tau_{R C}$ being the RC time scale of our cell at the beginning of charging phase) mainly to the parasitic current $\left(I_{p}\right)$ at that voltage. We show the parasitic current vs. capacitance voltage in the inset of Fig. 2b. We further made the assumption that parasitic current is only a function of voltage (not applied current) and used the relation below to calculate parasitic energy loss.

$$
E_{\text {in }}^{P}=\int_{0}^{t_{\text {charge }}} I_{p} V_{\text {cap }} d t \text { and } E_{\text {out }}^{P}=\int_{t_{\text {charge }}}^{t_{\text {cycle }}} I_{p} V_{\text {cap }} d t
$$

Note, as a visual aid, we have exaggerated the magnitudes of $E_{\text {in }}^{P}$ and $E_{\text {out }}^{P}$ in Fig. $2 \mathrm{~b}$ (although Fig. 2a is actual experimental data to scale). 
(a)
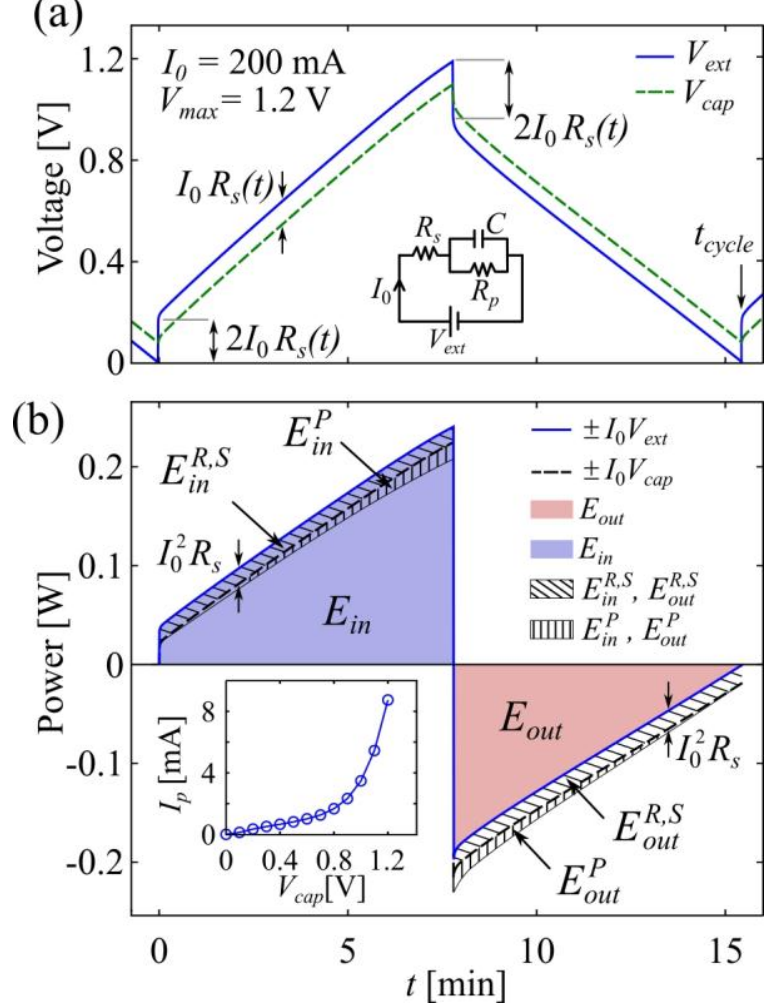

Fig. 2. (a) Measured voltage profile of the cell vs. time under $2 \mathrm{ml} \mathrm{min}^{-1}$ flow rate at $200 \mathrm{~mA}$ current and limit voltage of $V_{\max }=1.2 \mathrm{~V}$. Inset shows RC circuit analogy of the cell, where $R_{s}$ and $R_{p}$ respectively model series and parallel resistances in CDI. (b) Power input/generation of the cell for the conditions identical to those of (a). Shaded areas labeled as $E_{\text {in }}$ and $E_{\text {out }}$ show energy input and recovered during charging and discharging in a single cycle. Diagonal hatched areas show series resistive energy loss $\left(E_{\text {in }}^{R, S}\right.$ and $E_{\text {out }}^{R, S}$, and vertical hatched areas show parasitic energy losses $\left(E_{\text {in }}^{P}\right.$ and $E_{\text {out }}^{P}$ ). Inset shows measured parasitic current vs. $V_{\text {cap }}$ as obtained from independent, constant voltage experiments.

\subsection{In-situ series resistance measurement}


We performed in-situ, on-the-fly measurement of series resistance of the cell $\left(R_{s}\right)$ by sampling voltage response to a mid-frequency $(\sim 10 \mathrm{~Hz})$, small-amplitude $(2 \mathrm{~mA})$ AC current signal on top of the charging or discharging DC current $\left(I_{0}\right)$. We measured $R_{s}$ by dividing the measured voltage amplitude by current amplitude (c.f. Fig. S.4 of SI). $R_{s}$, as mentioned before, includes interfacial contact resistance and resistance of solution in spacers as well as external wires. For more information about resistance characterization refer to Section S.3 of the SI.

In Fig. 3, we show the results of series resistance measurements vs. capacitance voltage difference $\left(\Delta V_{c a p}\right)$ for currents of $25-300 \mathrm{~mA}$ and fixed limit voltage of $V_{\max }=1.2 \mathrm{~V}$. We define capacitance voltage difference as maximum variation of capacitance voltage during a full cycle. $\Delta V_{c a p}$ is defined as $V_{c a p, \max }-V_{\text {cap,min }}$. Each data point in each loop is an average of at least two measurements in two consecutive cycles under DSS conditions. The upper (lower) half of the loops corresponds to series resistance in the charging (discharging) process (see arrows in Fig. 3). As can be seen here, $R_{s}$ in the charging step is greater than that in the discharging step. This is because salt is removed from the spacers during charging. Cell operation under high currents therefore leads to greater asymmetry in resistance plots. This is expected, as charging with high currents removes a considerable portion of influent salt, which in turn, increases solution resistance in the spacer. As an example, Fig. S.2 of the SI shows more than $80 \%$ salt removed from inlet stream with $I_{0}=$ $300 \mathrm{~mA}$. Note that series resistance in this case varies by only about $30 \%$ (from 0.4 to $0.52 \Omega$ ). This suggests that interfacial (between electrodes and current collectors) and wire resistances contribute the majority of series resistance. A simple analysis (not shown here) suggests that spacer resistance is about $25 \%$ of total series resistance. We estimate resistance of titanium current collectors to be $<2 \%$ of $R_{s}$. In the inset of Fig. 3, we show the same resistance data vs. time 
(normalized by cycle time, $t_{\text {cycle }}$ ). The inset again shows a fast increase in resistance at higher currents. Refer to Section S.3 of the SI for a complete set of resistance plots. In Section 3.3, we will use these measurements to calculate resistive loss and present a comprehensive study of energy pathways in our fbCDI cell. To summarize, we list all the loss mechanisms we study in this work below.

i. Parasitic loss, $E_{\text {in }}^{P}+E_{\text {out }}^{P}$, calculated by Eq. (5). We hypothesize that this is primarily due to Faradaic currents.

ii. Resistive loss, $E_{\text {in }}^{R}+E_{\text {out }}^{R}$

ii.1. Series resistive loss, $E_{\text {in }}^{R, S}+E_{\text {out }}^{R, S}$, measured using $\sim 10 \mathrm{~Hz}$ probe signal and includes the following:

ii.1.1. Wires resistance

ii.1.2. Interfacial contact resistance between electrodes and current collectors

ii.1.3. Ionic resistance in spacers

ii.2. Non-series resistive loss, $E_{\text {in }}^{R, N S}+E_{\text {out }}^{R, N S}$, estimated as $\left(E_{\text {in }}-E_{\text {out }}\right)-\left(E_{\text {in }}^{P}+E_{\text {out }}^{P}\right)-$ $\left(E_{i n}^{R, S}+E_{\text {out }}^{R, S}\right)$. The primary component of this is the ionic resistance of solution within electrode pores. Note we neglect the distributed resistance within the electrode matrix (carbon) material. 


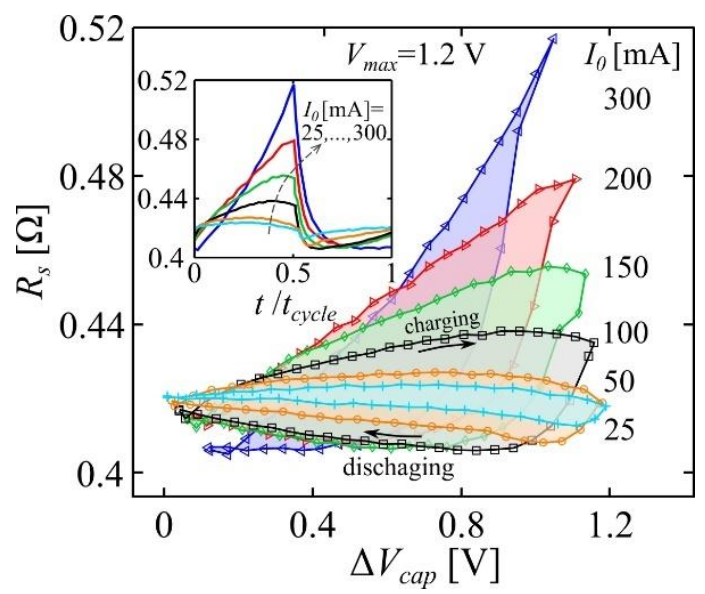

Fig. 3. Measured series resistance vs. $\Delta V_{\text {cap }}$ during charging and discharging for 25, 50, 100, 150, 200, and $300 \mathrm{~mA}$ currents and $V_{\max }=1.2 \mathrm{~V}$ (each loop corresponds to a fixed current). At low currents, $R_{s}$ does not vary considerably throughout the cycle, while it varies more strongly at high currents due to significant salt removal. The inset presents series resistance data vs. time (normalized by cycle time $t_{c y c l e}$ ) for one cycle.

\subsection{Energy losses in CDI}

In Fig. 4a, we show total energy loss per cycle $\left(E_{\text {in }}-E_{\text {out }}\right)$ vs. $\Delta V_{\text {cap }}$ for currents between 25$300 \mathrm{~mA}$. At a fixed current, energy loss monotonically increases with $\Delta V_{\text {cap }}$ (or equivalently, with cycle time). In Fig. S.7 of the SI, we show that cycle time increases almost linearly with $\Delta V_{\text {cap }}$. We also include $E_{\text {in }}$ and $E_{\text {out }}$ vs. $\Delta V_{\text {cap }}$ in Fig. S.7. Fig. 4a further shows that energy loss is generally greater at higher charging currents. We attribute this to the importance of the resistive loss which is approximately linearly proportional to current (for fixed charge transferred), and dominates the total loss at higher charging currents. We will discuss this in more detail below.

Fig. $4 \mathrm{~b}$ shows calculated parasitic loss $\left(E_{\text {in }}^{P}+E_{\text {out }}^{P}\right)$ vs. $\Delta V_{\text {cap }}$. Reduction of dissolved oxygen at $0.69 \mathrm{~V}$ (vs. SHE) and oxidation of carbon electrode at 0.7-0.9 $\mathrm{V}$ are considered as two main 
sources of parasitic reactions at voltages below electrolysis potential in CDI (He et al., 2016; Lee et al., 2010). We observe an exponential relation between parasitic loss and $\Delta V_{\text {cap }}$ (in the inset of this figure we plot the data on a logarithmic scale). This exponential growth is consistent with power loss due to parasitic reactions on the carbon surface. As given by the Butler-Volmer equation, the currents for these reactions (e.g. oxidation of surface groups and dissolved gasses in solution such as oxygen) are usually exponential with respect to surface potential. For example, Biesheuvel et al. (2012) used generalized Frumkin-Butler-Volmer model to derive an exponential relation between rate of redox reactions and Stern potential. Our results further show that parasitic loss is smaller at higher charging currents. For example, at $\Delta V_{\text {cap }} \approx 1.2 \mathrm{~V}$, parasitic loss at $100 \mathrm{~mA}$ is about 5 times smaller than the $25 \mathrm{~mA}$ case. We attribute this to the effect of series resistance voltage drop and cycle time. At high currents, cycle time is shorter and voltage drop across series resistances can be significant (c.f. Fig. S.1 of SI). So, the electrodes experience lower voltages (compared to low current cases) for a shorter period of time.

We show calculated resistive loss per cycle vs. $\Delta V_{\text {cap }}$ for 25-300 mA currents in Fig. 4c. As discussed in Section 2.2, to arrive at resistive loss, we first independently measured energy loss $\left(E_{\text {in }}-E_{\text {out }}\right)$, series resistive loss $\left(E_{\text {in }}^{R, S}+E_{\text {out }}^{R . S}\right)$, and parasitic loss $\left(E_{\text {in }}^{P}+E_{\text {out }}^{P}\right)$. We next used Eq. (1) to estimate energy loss due to non-series resistances $\left(E_{\text {in }}^{R, N S}+E_{\text {out }}^{R, N S}\right)$. We finally used Eqs. (3) and (4) to approximate resistive loss $\left(E_{\text {in }}^{R}+E_{\text {out }}^{R}\right)$. Results indicate that resistive loss increases proportionally with $\Delta V_{\text {cap }}$, or equivalently, with charging time (c.f. Fig. S.7 of SI). Fig. 4c also shows that resistive loss increases almost linearly with charging current.

Fig. $4 \mathrm{~d}$ shows calculated stored energy in the cell vs. $\Delta V_{\text {cap }}$. We have here made an assumption that non-series resistive losses during charging and discharging are approximately 
equal $\left(E_{\text {in }}^{R, N S} \approx E_{\text {out }}^{R, N S}\right)$ and so here approximate capacitance energy as $E_{\text {cap }} \approx E_{\text {in }}-\left[E_{\text {in,series }}^{R}+\right.$ $\left.\frac{1}{2}\left(E_{\text {in,ionic }}^{R}+E_{\text {out }, \text { ionic }}^{R}\right)\right]-E_{\text {in }}^{N R}$. We note that this approximation can be justified at low applied currents (where non-series resistance during charging and discharging should be almost equal), whereas at higher currents, it likely overpredicts $E_{c a p}$. Results show the collapse of data to a single quadraticform relation between $E_{c a p}$ and $\Delta V_{\text {cap }}$ in form of $E_{\text {cap }}=\frac{1}{2} C \Delta V_{\text {cap }}^{2}$ with $C \approx 110 \mathrm{~F}$ (or $26 \mathrm{~F} \mathrm{~g}^{-1}$ after normalizing by total electrode mass). The calculated value of $E_{c a p}$ here, although approximate, provides insight into energy efficiency of our CDI cell as we will discuss in the next section. To summarize, we here showed that energy losses in CDI have at least two components. First, there is an approximately linear (with voltage and/or current) resistive component (since resistive power scales as the square of current while cycle time is inverse to applied current). Second, there is an exponential (with voltage) parasitic component, and this is likely associated with parasitic reactions. We next turn our attention to the relative magnitude of these loss mechanisms. 

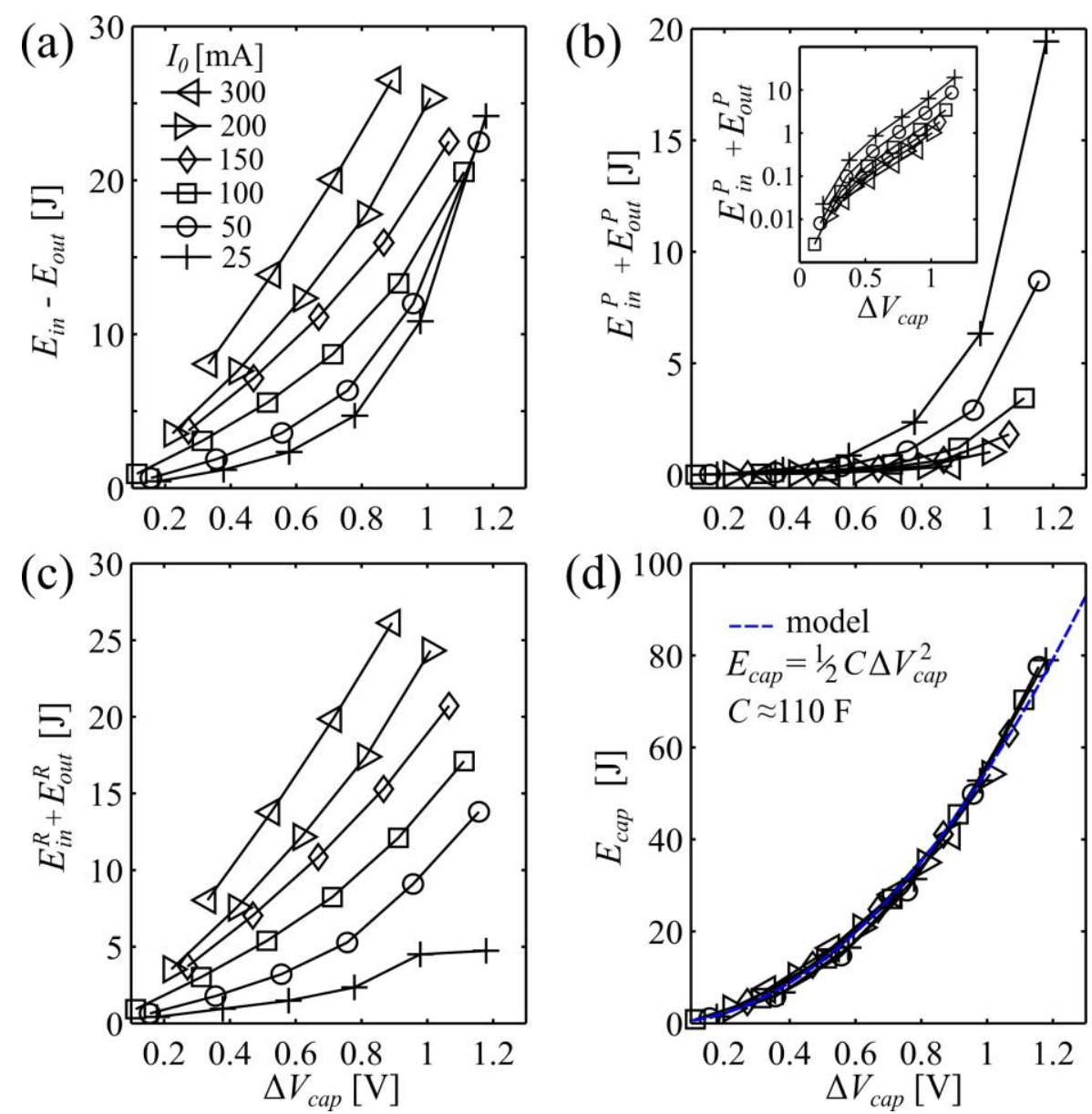

Fig. 4. (a) Measured energy loss per cycle vs. $\Delta V_{\text {cap }}$ for 25, 50, 100, 150, 200, and $300 \mathrm{~mA}$ currents. Energy loss increases with both $\Delta V_{\text {cap }}$ and $I_{0}$. At low currents, energy loss varies approximately exponential with $\Delta V_{c a p}$, while it is almost linear at high currents. (b) Measured parasitic loss per cycle vs. $\Delta V_{\text {cap }}$. Parasitic losses (likely associated with Faradaic reactions) vary exponentially with $\Delta V_{\text {cap }}$ (see inset). (c) Resistive loss (series and non-series) in one cycle for experimental conditions identical to those of (a). Resistive loss increases almost linearly with both $\Delta V_{\text {cap }}$ and $I_{0}$. (d) Calculated stored energy is well described as the square of $\Delta V_{c a p}$. 
Fig. 5a demonstrates the relative importance of energy loss mechanisms by plotting the ratio of (series plus non-series) resistive loss to total energy loss in one cycle vs. $\Delta V_{c a p}$ for currents ranging from 25 to $300 \mathrm{~mA}$. The shaded and white areas respectively correspond to parasitic dominant (> 50\% parasitic) and resistive dominant (> 50\% resistive) conditions. The results show that the resistive energy loss dominates the total loss at high charging current and small $\Delta V_{c a p}$ cases. Both resistive and parasitic losses decrease with decreasing $\Delta V_{\text {cap }}$, but the exponential dependence of parasitic loss on $\Delta V_{c a p}$ makes it negligible at low $\Delta V_{c a p}$. Parasitic loss, however, is dominant at low current and high $\Delta V_{\text {cap }}$ (see shaded area where parasitic $>50 \%$ of total loss). This is because, as Figs. 4b and 4c suggest, resistive loss linearly increases with current, while parasitic loss generally decreases with charging current.

In Fig. 5b, we show the ratio of capacitor energy, $E_{c a p}$, over total energy loss in a cycle vs. $\Delta V_{c a p}$ for the applied current values of Fig. 5a. This ratio is essentially an energy transfer coefficient and reflects the efficiency of energy storage in the cell. As a visual aid, the shaded region is plotted to be consistent with Fig. 5a. Results show that this ratio is generally greater at lower charging currents. However, in the lowest charging currents (i.e. 25 and $50 \mathrm{~mA}$ ), we observe a maximum at a voltage in which parasitic and resistive losses are comparable. We hypothesize that this optimum operating point balancing resistive and parasitic losses may hold for other CDI systems, at least for low to moderate applied current densities, although more evidence is needed before we can confirm this. 

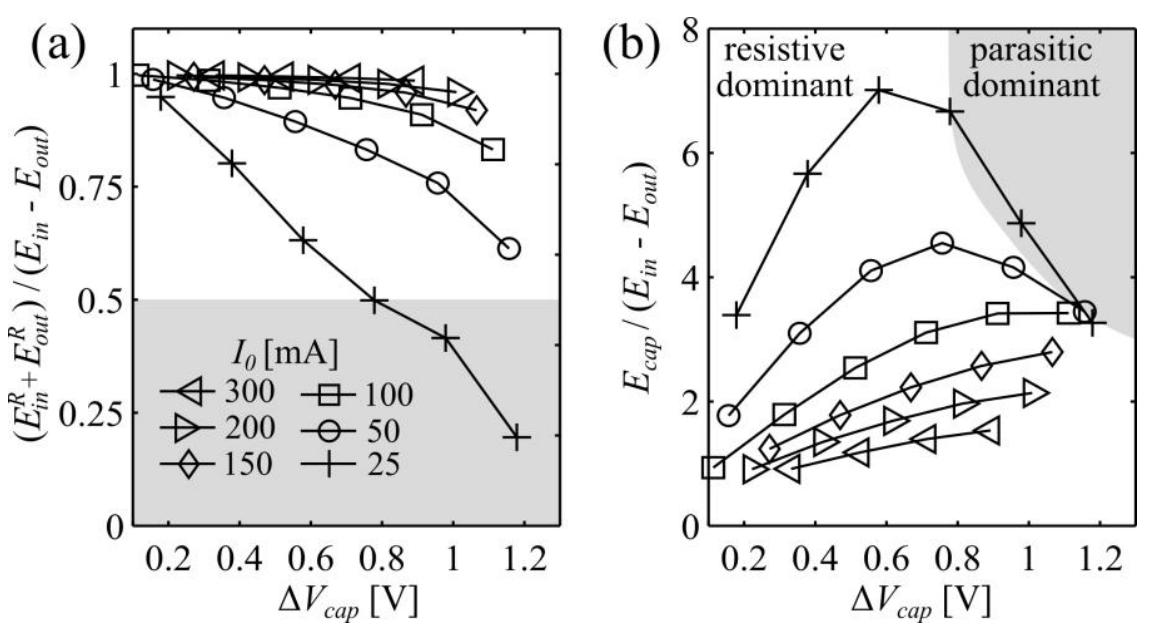

Fig. 5. (a) Ratio of resistive to total energy loss in one cycle vs. $\Delta V_{\text {cap }}$ for 25-300 mA currents.

Resistive loss dominates total loss at high charging current and small $\Delta V_{\text {cap }}$ cases. Parasitic loss, however, is dominant at low current and high $\Delta V_{\text {cap }}$ (see shaded area in which parasitic $>50 \%$ of total loss). (b) Ratio of stored charge to total energy loss in one cycle vs. $\Delta V_{\text {cap }}$ for the same data as in (a). This ratio quantifies the effectiveness of energy storage in the cell and is generally greater at lower currents. Results show this ratio has an optimum at small currents (25 and $50 \mathrm{~mA}$ ), and this optimum coincides with $\Delta V_{\text {cap }}$ at which (series plus non-series) resistive loss and parasitic loss are comparable.

\subsection{Energy and salt adsorption performance in CDI}

We here present two performance FOMs for our cell. The first metric is average salt adsorption rate $(A S A R)$ in units of moles of salt per total electrode area per time and can be defined as (Suss et al., 2015)

$$
A S A R=\frac{\Gamma_{a d s}}{N A t_{\text {cycle }}}=\frac{Q}{N A t_{\text {cycle }}} \int_{0}^{t_{\text {charge }}}\left(c-c_{0}\right) \mathrm{d} t
$$


where $\Gamma_{a d s}$ is amount of salt adsorbed during charging (in units of moles), $N=5$ is number for electrode pairs, $A \approx 28 \mathrm{~cm}^{2}$ is single electrode area, $t_{\text {cycle }}$ is cycle time, $t_{\text {charge }}$ is charging time, $Q$ is flow rate, and $c$ and $c_{0}$ are effluent and influent salt concentrations, respectively. This metric quantifies the throughput of the desalination process. Second is energy normalized adsorbed salt ( $E N A S$ ) in units of moles of salt per Joules of energy lost and is defined as

$$
E N A S=\frac{Q \int_{0}^{t_{\text {charge }}}\left(c-c_{0}\right) \mathrm{d} t}{E_{\text {in }}-E_{\text {out }}},
$$

which quantifies the energetic efficiency of the desalination process. In Fig. 6, we show values of $\Gamma_{\text {ads }}$ (in units of $\mu$ mole $\mathrm{cm}^{-2}$ and $\mathrm{mg} \mathrm{g}^{-1}$ ), ASAR (in units of $\mu$ mole $\mathrm{cm}^{-2} \mathrm{~min}^{-1}$ and $\mathrm{mg} \mathrm{g}^{-1} \mathrm{~min}^{-1}$ ), and ENAS (in units of $\mu$ mole $\mathrm{J}^{-1}$ and $\mathrm{mg} \mathrm{J}^{-1}$ ) vs. $\Delta V_{\text {cap }}$ for various currents mentioned before. The conversion between our two forms of normalization can be performed by using a total mass of the ten individual electrodes of $4.3 \mathrm{~g}$, an area of $A \approx 28 \mathrm{~cm}^{2}$ per electrode, and the $\mathrm{KCl}$ atomic mass of $\left.74.55 \mathrm{~g} \mathrm{~mole}^{-1}\right)$. We have here normalized $\Gamma_{\text {ads }}$ and $A S A R$ by stack electrode area (NA) and total electrode mass. Fig. 6a shows that charging the cell with higher $\Delta V_{\text {cap }}$ leads to greater salt adsorption. Operating at lower charging current generally has the same effect. Salt adsorption, however, can decrease for very low currents of $25 \mathrm{~mA}$ and high $\Delta V_{\text {cap }}$ (where parasitic loss dominates). For example, at fixed $\Delta V_{c a p} \approx 1.2 \mathrm{~V}$, salt adsorption at $25 \mathrm{~mA}$ current results in significant charge consumed by parasitic losses, and this results in less salt adsorbed than the $50 \mathrm{~mA}$ case.

$A S A R$ and ENAS are. 6b and 6c. Regions with dominant parasitic loss are indicated by grey shading. In resistive shown in Figs dominant regimes, both $A S A R$ and ENAS increase with $\Delta V_{\text {cap }}$, however, as parasitic loss becomes dominant, ASAR and ENAS can decrease with $\Delta V_{\text {cap }}$. 
An important observation in Fig. 6a is the inefficiency of salt adsorption at high currents. That is, ASAR does not noticeably improve from 200 to $300 \mathrm{~mA}$ current. We attribute this retardation of salt removal rate to the relative magnitude of cell time constant (defined as ratio of cell volume to flow rate) and charging time $\left(t_{\text {charge }}\right)$. In Appendix A, we develop a simple transport model for effluent salt concentration under $\mathrm{CC}$ charging condition and show that the time scale for concentration to reach a plateau can be well described by a simple cell time constant of the form $t_{\text {cell }}=v_{\text {cell }} / Q\left(v_{\text {cell }}\right.$ and $Q$ being cell volume and flow rate, respectively). At high currents (beyond $200 \mathrm{~mA}$ ), the charging time $t_{\text {charge }}$ is so short (on the order of a few minutes) that it becomes comparable to $t_{\text {cell }}$. As a result, the charging phase finishes "prematurely" (discharge phase starts before effluent concentration reaches its plateau level). This is evident for the case of $300 \mathrm{~mA}$ charging current and limit voltage of $V_{\text {ext }}=1.2 \mathrm{~V}$, where the shape of the effluent concentration profile has no clear plateau region (c.f. Fig. A.1). Another possible reason is the effect of readsorption of desorbed salt from previous discharging phase. This is specifically problematic at high currents, as the flush time and charging time are on the same order.
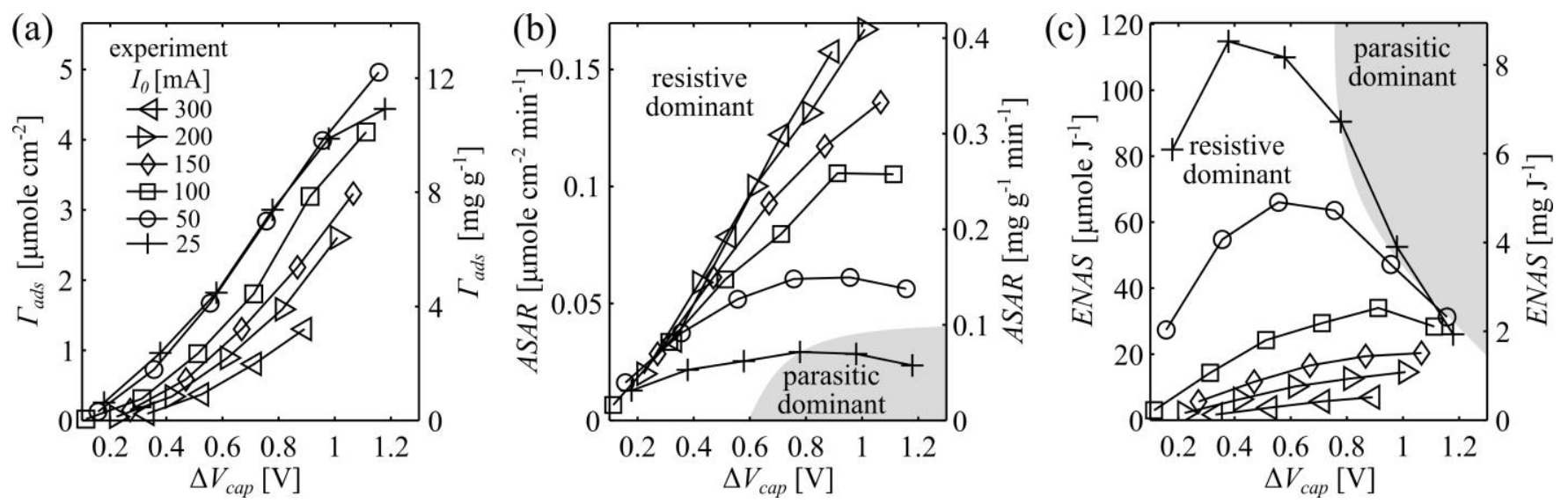
Fig. 6. (a) Normalized salt adsorption $\left(\Gamma_{a d s}\right)$ in units of $\mu$ mole $\mathrm{cm}^{-2}$ and $\mathrm{mg} \mathrm{g}^{-1}$, (b) average salt adsorption rate $(A S A R)$ in units of $\mu$ mole $\mathrm{cm}^{-2} \mathrm{~min}^{-1}$ and $\mathrm{mg} \mathrm{g}^{-1} \mathrm{~min}^{-1}$, and (c) energy normalized adsorbed salt ( ENAS ) in units of $\mu$ mole $\mathrm{J}^{-1}$ and $\mathrm{mg} \mathrm{J}^{-1}$, each as a function of $\Delta V_{\text {cap }}$. Results are for the experimental conditions identical to those of Fig. 4. The interplay between resistive effects and parasitic effects results in maxima in $A S A R$ and ENAS for low-to-midrange applied currents.

For a better representation of $A S A R$ and ENAS results, in Figs. 7a and 7b, we show interpolated contour plots of these two metrics as functions of $\Delta V_{c a p}$ and external current for the same data as in Figs. 6b and 6c. The markers overlaid on the contour plots are the corresponding measurement points (at each current and voltage). The dashed curves are consistent with those of Figs. 5 and 6 and indicate the locus of equal resistive and parasitic losses. As discussed earlier, $A S A R$ increases as either current or $\Delta V_{\text {cap }}$ increases. However, as current exceeds $200 \mathrm{~mA}$, $A S A R$ remains constant or even decreases at low $\Delta V_{\text {cap }}$. ASAR results, therefore, show the best removal rate performance at mid-level currents $(\sim 200 \mathrm{~mA})$ and highest possible $\Delta V_{\text {cap }}(\sim 1.1 \mathrm{~V})$. In contrast, Fig. 7b shows that ENAS (indicator of energetic performance) is maximized at lower currents and mid-level voltage $(\sim 0.6 \mathrm{~V})$. Note that, similar to our observations of the data of Fig. 6c, ENAS rapidly drops (with increasing $\Delta V_{\text {cap }}$ ) as parasitic losses begin to dominate the energy loss. This suggests that there is no operational point that simultaneously favors the two performance requirements considered, namely, removal rate and low energy cost. To elaborate this, we plot ENAS versus $A S A R$ for different external currents in Fig. 7c. Data points in each curve correspond to a variation in the value of $\Delta V_{\text {cap }}$ (as shown in Figs. 6b and 6c). The results 
clearly show a tradeoff between removal rate and energy efficiency of desalination process ( ASAR and ENAS respectively quantify desalination speed and energetic performance of the cell). For example, small charging currents are generally more favorable in terms of energy performance (higher $E N A S$ ), while large currents have higher adsorption rate (higher ASAR). It is possible, however, to combine ASAR and ENAS into a new metric (or user-defined cost function) and optimize the resulting metric. In Section S.5 of the SI, we introduce an energetic operational metric (EOM) as the product of $A S A R$ and ENAS and seek a combination of current and $\Delta V_{\text {cap }}$ which maximizes our EOM. Interestingly, we show that the location of maximum EOM approximately coincides with the locus of operational points where (series plus non-series) resistive loss and parasitic loss are comparable.
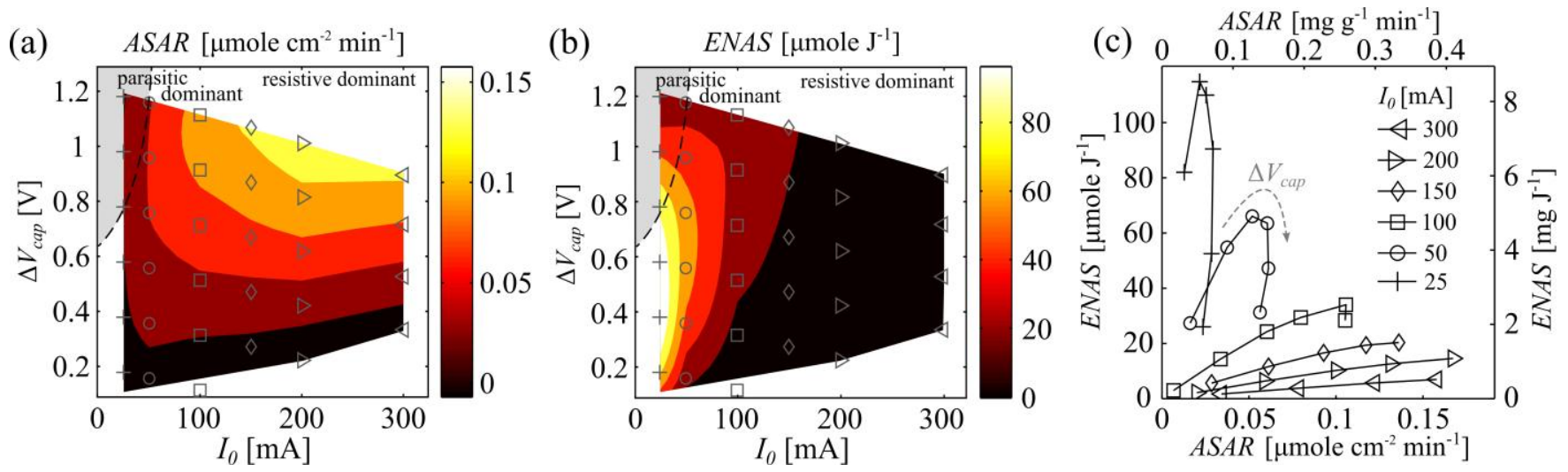

Fig. 7. Contour plots of interpolated (a) average salt adsorption rate (ASAR) in units of $\mu$ mole $\mathrm{cm}^{-2} \mathrm{~min}^{-1}$ and (b) energy normalized adsorbed salt ( $E N A S$ ) in units of $\mu$ mole $\mathrm{J}^{-1}$ vs. current $I_{0}$ and $\Delta V_{\text {cap }}$. (c) $A S A R$ versus $E N A S$ for the same data as in (a) and (b). The arrow shows direction of increase of $\Delta V_{\text {cap }}$. ASAR and ENAS respectively quantify desalination speed and energetic performance of the cell. Results show a tradeoff between the two: ASAR is greatest at high currents and high $\Delta V_{c a p}$, while ENAS is generally greater in low currents and low $\Delta V_{\text {cap }}$. In 
very low currents (i.e. 25 and $50 \mathrm{~mA}$ ), however, ENAS shows an abrupt drop as $\Delta V_{\text {cap }}$ passes a certain limit. The optimum value of ENAS at lowest currents corresponds to where resistive and parasitic losses are comparable.

\section{Conclusions}

We have quantified individual loss mechanisms operative during CDI charging and discharging, and characterized their dependence on the parameters of charging current and maximum cell voltage. We identified losses dependent on cell voltage attributable to parasitic currents and losses depending on charging rate, which are dominated by cell resistances. We measured series resistance for the cell throughout charge/discharge phases for a range of input solute concentrations and a variety of charging currents and cell voltages. We also used independent experiments to quantify parasitic losses as a function of voltage in double layers. The two categories of loss favor different charging rates, with resistive losses minimized at low charging currents, but parasitic losses (and associated leakage current losses) lessened for higher rates which reduce the time the

cell spends at high voltage. We introduced two figures of merit, ASAR and ENAS, which characterize the performance of a CDI cell in terms of throughput and energy efficiency, respectively. We showed that these figures of merit provide a powerful tool for optimizing CDI operation.

\section{Acknowledgements}


This work was supported jointly by LLNL LDRD project 15-ERD-068 and TomKat Center for Sustainable Energy at Stanford University. Work at LLNL was performed under the auspices of the US DOE by LLNL under Contract DE-AC52-07NA27344. A.H. gratefully acknowledges the support from the Stanford Graduate Fellowship program of Stanford University. J.G.S. and A.H. also gratefully acknowledge support from TomKat Center for Sustainable Energy as part of the Distributed Production and Energy Generation program. The authors would like to thank Maarten Biesheuvel of Wetsus, the Netherlands' Centre of Excellence for Sustainable Water Technology for providing the electrode materials.

\section{Appendix A. Time scales in fbCDI}

We here present a simple transport model for fbCDI systems under constant current and constant flow rate operation and identify relevant time scales. Starting with a one-dimensional transport equation for salt along the flow direction and neglecting diffusion, we have

$$
p_{s p} \frac{\partial c}{\partial t}+u_{s u p} \frac{\partial c}{\partial x}=\frac{\Lambda}{F} i
$$

where $p_{s p}$ is porosity of the spacer, $u_{s u p}$ is superficial velocity in the spacer (by definition the

product of $u_{s u p}$, spacer area perpendicular to the flow, and spacer porosity $p_{s p}$ is the volume flow rate), $i$ is the current density, $\Lambda$ is charge efficiency, and $F$ is Faraday's constant. We assume a constant inlet concentration $c_{0}$, fixed charge efficiency, and uniform (transverse) current density along the flow. We then integrate Eq. (A.1) in the direction of flow and arrive at

$$
p_{s p} \frac{\partial c_{\text {out }}}{\partial t}=\frac{Q}{v_{\text {cell }}}\left(c_{0}-c_{\text {out }}\right)-\frac{\Lambda}{F} I_{0}
$$


where $v_{\text {cell }}$ is cell volume (volume of spacers), $c_{\text {out }}$ is effluent concentration, $Q$ is flow rate, and $I_{0}$ is applied current. Equation (2) can be written as

$$
\frac{\partial \bar{c}_{\text {out }}}{\partial t}=\bar{Q}\left(1-\bar{c}_{\text {out }}\right)-\bar{I}_{0},
$$

where $\bar{c}_{\text {out }}=c_{\text {out }} / c_{0}, \bar{Q}=Q /\left(p_{s p} v_{\text {cell }}\right)$, and $\bar{I}_{0}=\Lambda I_{0} /\left(F p_{s p} v_{\text {cell }} c_{0}\right)$. Effluent concentration can then be solved as

$$
\bar{c}_{\text {out }}=1-\frac{\bar{I}_{0}}{\bar{Q}}[1-\exp (-\bar{Q} t)]
$$

This simplified analysis shows effluent concentration profile should exhibit a time scale of $\tau_{\text {cell }}=1 / \bar{Q}$. We show the result of normalized effluent concentration in Fig. A.1a. Time scale for concertation to reach a plateau level is $1 / \bar{Q}$, and (normalized) concentration change under constant current charging $I_{0}$ condition is $\Delta \bar{c}=\bar{I}_{0} / \bar{Q}$. The second time scale shown in Fig. A.1a is charging time $\tau_{\text {charge }}$, which can be approximated by the ideal capacitor equation as $\tau_{\text {charge }}=C \Delta V_{\text {cap }} / I_{0}$. Equating the two time scales gives a linear relation between current and flow rate as $Q / I=$ $p_{s p} v_{\text {cell }} /\left(C \Delta V_{\text {cap }}\right)$. Fig. A.1b is then a regime map constructed by plotting flow rate versus current (in logarithmic scale). This figure summarizes the two possible regimes based on relative values of $\tau_{\text {cell }}$ and $\tau_{\text {charge }}$. The upper-left region corresponds to a "plateau mode" regime, where the effluent concentration reaches a steady level before the charging phase ends. The lower-right regime corresponds to a "triangular-peaked" regime, where the charging phase ends prematurely, and the effluent does not reach to a plateau. 

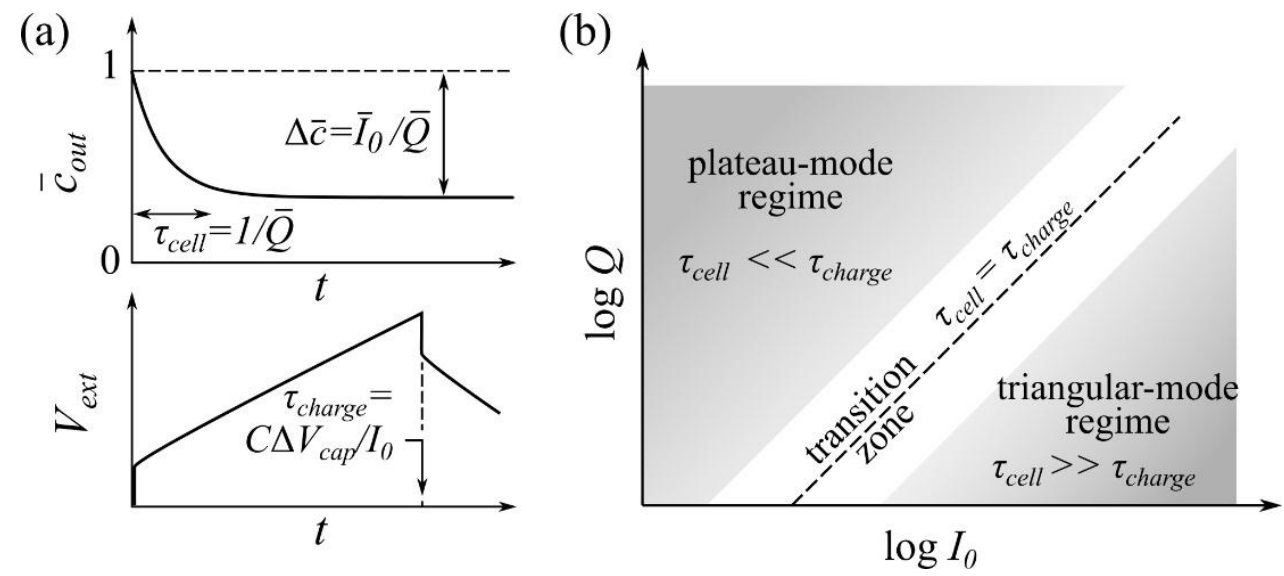

Fig. A.1. (a) Schematic of effluent concentration profile and voltage profile for an fbCDI cell under constant current and constant flow rate conditions. (b) Regime map corresponding to "plateau" mode (upper-left region) with low current and high flow rate, and "triangular" mode (lower-right region) with relatively high current and low flow rate.

\section{References}

Alvarez-Gonzalez, F.J., Martin-Ramos, J.A., Diaz, J., Martinez, J.A., Pernia, A.M., 2016. EnergyRecovery Optimization of an Experimental CDI Desalination System. IEEE Trans. Ind. Electron. doi:10.1109/TIE.2015.2494015

Anderson, M. a., Cudero, A.L., Palma, J., 2010. Capacitive deionization as an electrochemical means of saving energy and delivering clean water. Comparison to present desalination practices: Will it compete? Electrochim. Acta 55, 3845-3856. doi:10.1016/j.electacta.2010.02.012

Biesheuvel, P.M., 2015. Activated carbon is an electron-conducting amphoteric ion adsorbent 19.

Biesheuvel, P.M., Fu, Y., Bazant, M.Z., 2012. Electrochemistry and capacitive charging of porous 
electrodes in asymmetric multicomponent electrolytes. Russ. J. Electrochem. 48, 580-592. doi:10.1134/S1023193512060031

Biesheuvel, P.M., Hamelers, H.V.M., Suss, M.E., 2016. Theory of Water Desalination by Porous Electrodes with Immobile Chemical Charge. Colcom 9, 1-5. doi:10.1016/j.colcom.2015.12.001

Black, J., Andreas, H. a., 2010. Prediction of the self-discharge profile of an electrochemical capacitor electrode in the presence of both activation-controlled discharge and charge redistribution. J. Power Sources 195, 929-935. doi:10.1016/j.jpowsour.2009.08.040

Black, J., Andreas, H. a., 2009. Effects of charge redistribution on self-discharge of electrochemical capacitors. Electrochim. Acta 54, 3568-3574. doi:10.1016/j.electacta.2009.01.019

Blair, J.W., Murphy, G.W., 1960. Electrochemical Demineralization of Water with Porous Electrodes of Large Surface Area, in: Saline Water Conversion, Advances in Chemistry. American Chemical Society, pp. 20-206. doi:doi:10.1021/ba-1960-0027.ch020

Choi, J.-H., 2015. Comparison of constant voltage (CV) and constant current (CC) operation in the membrane capacitive deionisation process. Desalin. Water Treat. 56, 921-928. doi:10.1080/19443994.2014.942379

Cohen, I., Avraham, E., Bouhadana, Y., Soffer, A., Aurbach, D., 2015. The effect of the flowregime, reversal of polarization, and oxygen on the long term stability in capacitive deionization processes. Electrochim. Acta 153, 106-114. doi:10.1016/j.electacta.2014.12.007

Conway, B.E., 2013. Electrochemical supercapacitors: scientific fundamentals and technological applications. Springer Science \& Business Media.

Conway, B.E., Pell, W.G., 2002. Power limitations of supercapacitor operation associated with 
resistance and capacitance distribution in porous electrode devices. J. Power Sources 105, 169-181. doi:10.1016/S0378-7753(01)00936-3

Demirer, O.N., Naylor, R.M., Rios Perez, C.A., Wilkes, E., Hidrovo, C., 2013. Energetic performance optimization of a capacitive deionization system operating with transient cycles and brackish water. Desalination 314, 130-138. doi:10.1016/j.desal.2013.01.014

Długołęcki, P., van der Wal, A., 2013. Energy recovery in membrane capacitive deionization. Environ. Sci. Technol. 47, 4904-10. doi:10.1021/es3053202

Dykstra, J.E., Dijkstra, J., van der Wal, A., Hamelers, H.V.M., Porada, S., 2016. On-line method to study dynamics of ion adsorption from mixtures of salts in capacitive deionization. Desalination 390, 47-52. doi:10.1016/j.desal.2016.04.001

Dykstra, J.E., Zhao, R., Biesheuvel, P.M., Van der Wal, A., 2016. Resistance identification and rational process design in Capacitive Deionization. Water Res. 88, 358-370. doi:10.1016/j.watres.2015.10.006

Elimelech, M., Phillip, W.A., 2011. The Future of Seawater and the Environment: Energy, Technology, and the Environment. Science (80-. ). 333, 712-718. doi:10.1126/science. 1200488

García-Quismondo, E., Santos, C., Lado, J., Palma, J., Anderson, M.A., 2013. Optimizing the energy efficiency of capacitive deionization reactors working under real-world conditions. Environ. Sci. Technol. 47, 11866-11872. doi:10.1021/es4021603

García-quismondo, E., Santos, C., Soria, J., Palma, J., Anderson, M.A., 2015. New Operational Modes to Increase Energy Efficiency in $\mathrm{Ca}$ - pacitive Deionization Systems. doi:10.1021/acs.est.5b05379

He, D., Wong, C.E., Tang, W., Kovalsky, P., Waite, T.D., 2016. Faradaic Reactions in Water 
Desalination by Batch-Mode Capacitive Deionization. Environ. Sci. Technol. Lett. acs.estlett.6b00124. doi:10.1021/acs.estlett.6b00124

Hemmatifar, A., Stadermann, M., Santiago, J.G., 2015. Two-Dimensional Porous Electrode Model for Capacitive Deionization. J. Phys. Chem. C 119, 24681-24694. doi:10.1021/acs.jpcc.5b05847

Ike, I.S., Sigalas, I., Iyuke, S., 2016. Understanding performance limitation and suppression of leakage current or self-discharge in electrochemical capacitors: a review. Phys. Chem. Chem. Phys. 18, 661-680. doi:10.1039/C5CP05459A

Johnson, A.M., Newman, J., 1971. Desalting by Means of Porous Carbon Electrodes. J. Electrochem. Soc. 118, 510. doi:10.1149/1.2408094

Kang, J., Kim, T., Jo, K., Yoon, J., 2014. Comparison of salt adsorption capacity and energy consumption between constant current and constant voltage operation in capacitive deionization. Desalination 352, 52-57. doi:10.1016/j.desal.2014.08.009

Lee, J.-H.H., Bae, W.-S.S., Choi, J.-H.H., 2010. Electrode reactions and adsorption/desorption performance related to the applied potential in a capacitive deionization process. Desalination 258, 159-163. doi:10.1016/j.desal.2010.03.020

Pell, W.G., Conway, B.E., Marincic, N., 2000. Analysis of non-uniform charge/discharge and rate effects in porous carbon capacitors containing sub-optimal electrolyte concentrations. J. Electroanal. Chem. 491, 9-21. doi:10.1016/S0022-0728(00)00207-2

Qu, Y., Baumann, T.F., Santiago, J.G., Stadermann, M., 2015. Characterization of Resistances of a Capacitive Deionization System. Environ. Sci. Technol. 1-15. doi:10.1021/acs.est.5b02542

Rica, R. a., Ziano, R., Salerno, D., Mantegazza, F., Bazant, M.Z., Brogioli, D., 2013. Electrodiffusion of ions in porous electrodes for capacitive extraction of renewable energy from 
salinity differences. Electrochim. Acta 92, 304-314. doi:10.1016/j.electacta.2013.01.063

Shrivastava, A., Rosenberg, S., Peery, M., 2014. Energy efficiency breakdown of reverse osmosis and its implications on future innovation roadmap for desalination. Desalination 368, 181192. doi:10.1016/j.desal.2015.01.005

Suss, M., Porada, S., Sun, X., Biesheuvel, M., Yoon, J., Presser, V., 2015. Water desalination via capacitive deionization: what is it and what can we expect from it? Energy Environ. Sci. doi:10.1039/C5EE00519A

Suss, M.E., Baumann, T.F., Worsley, M. a., Rose, K. a., Jaramillo, T.F., Stadermann, M., Santiago, J.G., 2013. Impedance-based study of capacitive porous carbon electrodes with hierarchical and bimodal porosity. J. Power Sources 241, 266-273. doi:10.1016/j.jpowsour.2013.03.178

Yang, H., Zhang, Y., 2013. Analysis of supercapacitor energy loss for power management in environmentally powered wireless sensor nodes. IEEE Trans. Power Electron. 28, 53915403. doi:10.1109/TPEL.2013.2238683

Yu, A., Chabot, V., Zhang, J., 2013. Electrochemical supercapacitors for energy storage and delivery: fundamentals and applications. CRC Press.

Zhao, R., Biesheuvel, P.M., van der Wal, a., 2012. Energy consumption and constant current operation in membrane capacitive deionization. Energy Environ. Sci. 5, 9520. doi:10.1039/c2ee21737f

Zhao, R., Porada, S., Biesheuvel, P.M., van der Wal, a., 2013. Energy consumption in membrane capacitive deionization for different water recoveries and flow rates, and comparison with reverse osmosis. Desalination 330, 35-41. doi:10.1016/j.desal.2013.08.017

Zhao, R., van Soestbergen, M., Rijnaarts, H.H.M., van der Wal, A., Bazant, M.Z., Biesheuvel, P.M., 2012. Time-dependent ion selectivity in capacitive charging of porous electrodes. J. 
Colloid Interface Sci. 384, 38-44. doi:10.1016/j.jcis.2012.06.022 